
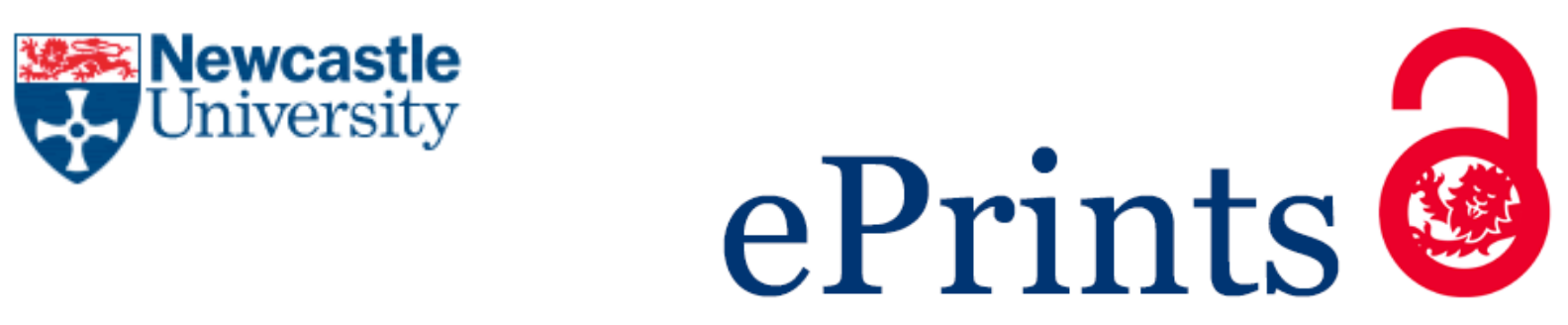

Salis C, Murray L, Bakas K.

An International Survey of Assessment Practices for Short-Term and Working

Memory Deficits in Aphasia.

American Journal of Speech-Language Pathology 2018

DOI: https://doi.org/10.1044/2017 AJSLP-17-0057

\title{
Copyright:
}

This is the authors' accepted manuscript of an article that was published in its final definitive form by American Speech-Language-Hearing Association, 2018.

DOI link to article:

https://doi.org/10.1044/2017 AJSLP-17-0057

Date deposited:

$27 / 10 / 2017$

Embargo release date:

20 August 2018

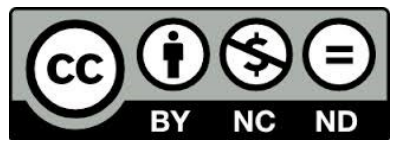

This work is licensed under a

Creative Commons Attribution-NonCommercial-NoDerivatives 4.0 International licence 


\section{An international survey of assessment practices for short-term and working memory deficits in aphasia}

Running head: survey of STM/WM memory assessment in aphasia Keywords: aphasia, assessment, memory

Declaration of interest: The authors declare no known conflict of interest.

Christos Salis (corresponding author)

Speech \& Language Sciences, Newcastle University

King George VI building, Queen Victoria Road

Newcastle upon Tyne, NE1 7RU, UK

christos.salis@ncl.ac.uk

tel. no. +441912088875

Laura Murray

School of Communication Sciences \& Disorders, Western University

Elborn College, London, Ontario, N6G 1H1, Canada

1murra57@uwo.ca

tel. no. +15196612111

Katrina Bakas

Communication Sciences and Disorders, University of Cincinnati

Cincinnati, OH 45219, USA

bakaskl@mail.uc.edu

tel. no. +13176904798 


\begin{abstract}
Purpose: Recent research has highlighted the clinical relevance of understanding the nature of short-term (STM) and working memory (WM) deficits in persons with aphasia and the way these deficits affect linguistic processing and functional communication in activities of daily living. The psychometric properties of tests commonly used to identify STM/WM problems in individuals with aphasia, however, have been questioned. No previous study has sought to investigate assessment practices and attitudes by speech-language pathologists involved in aphasia management. Accordingly, the aims of this study were: (a) to investigate both attitudes toward STM/WM assessment in individuals with aphasia as well as the types and frequency of STM/WM tests used with individuals with aphasia; and (b) to explore factors (e.g., educational background) that may influence STM/WM assessment practices.
\end{abstract}

Method: Respondents recruited via professional and aphasia support organizations completed an on-line survey. The survey elicited information about the respondents' demographic and clinical backgrounds, STM/WM assessment clinical practices and views, including frequency and preferred use of specific STM/WM tests.

Results: The majority of respondents reported regular use of STM/WM tests as part of aphasia management. Positive attitudes towards STM/WM assessments were also reported. The most popular rankings of tests were the Cognitive-Linguistic Quick Test, the Comprehensive Aphasia Test, and the Token Test. Results suggested limited knowledge about measures that assess selfperceptions of functional memory abilities. Regression analyses showed that the frequency of reported STM/WM test use was similar between clinicians and dual role researchers/clinicians, 
but their attitudes towards the value of STM/WM differed. USA and UK respondents reported similar assessment practices.

Conclusions: It is reassuring that STM/WM is taken into consideration by clinicians when providing aphasia management. Two of the most popular tests, however, have poor psychometric properties and caution should be exercised in clinical decision-making. The different value placed on STM/WM testing by clinicians and researchers/clinicians has implications for continuing professional development. 


\section{Introduction}

Impairments of short-term and working memory functioning, both verbal and non-verbal, are prevalent among individuals with aphasia, regardless of the severity or type of their aphasia (e.g., Lang \& Quitz, 2012; Mayer \& Murray, 2012). Although related, these memory subsystems differ (Baddeley, 2012). Short-term memory (STM) supports the brief storage of information in a nominally processed state. In contrast, working memory (WM) allows not only temporarily storing information, but also manipulating that information to meet a specific objective. STM and WM are similarly viewed as capacity-limited systems because each can retain only a restricted amount of information for a restricted period of time. Another distinguishing feature in the two memory systems is how they are measured. STM measures are considered simple (e.g., serial recall of words), whereas WM measures are considered complex (e.g., alphabet span in which a series of presented words must be rearranged to recall in alphabetical order) with typically linguistically complex test/task instructions (cf., Conway, Cowan, Bunting, Therriault, Scott, \& Minkoff, 2002). In terms of the neural correlates, there is some overlap between regions and circuits supporting not only STM and WM, but also language abilities (Cahana-Amitay \& Albert, 2015; Murray \& Mayer, 2016). For instance, a meta-analysis of studies involving healthy individuals (Rottschy et al., 2012) reported a widespread bilateral fronto-parietal network for WM, with several regions sensitive to specific task components. For example, Broca's region was selectively active during verbal tasks whereas ventral and dorsal premotor cortices were preferentially involved in memory for object identity and location, respectively. Likewise, studies involving persons with aphasia have found that auditory-verbal STM is supported by regions in the left temporo-parietal cortex (e.g., Baldo, Katseff \& Dronkers, 2012), a cortical region also crucial for language comprehension. Accordingly, brain damage that results in 
language impairments such as aphasia is likely to additionally produce STM and WM difficulties (Cahana-Amitay \& Albert, 2015; Murray \& Mayer, 2016).

Indeed, the study of STM/WM status in aphasia has had a long history (e.g., Eling, 2015), with the overwhelming majority of studies seeking to explicate the interface between memory and language by examining relationships between STM/WM and core aspects of linguistic processing including phonological, lexical-semantic, syntactic, and discourse (e.g., Caplan, Michaud, \& Hufford, 2013). Such research has led to staunch theoretical claims about the underlying nature of linguistic deficits in aphasia, either as causative or secondary impairments of auditory-verbal STM (Martin \& Ayala, 2004; Schuell, Jenkins, \& Jiménez-Pabón, 1964), as well as, sub-impairments of verbal STM alongside phonological and semantic fault lines (Martin \& Allen, 2008; Martin \& Ayala, 2004).

Beyond such theoretical debates, contemporary researchers have shifted to examine clinical implications and applications (Murray, 2012; Salis, Kelly, \& Code, 2015). In particular, there is growing recognition that STM/WM abilities play a role in aphasia recovery. For example, Lang and Quitz (2012) identified that in the subacute recovery stage, there is a memory gradient worsening progressively from verbal to non-verbal STM/WM, with more severe aphasia associated with more impaired memory skills. Other research has suggested a potent relationship between STM/WM abilities and language treatment outcomes. Relatedly, Harnish and Lundine (2015) reported that visuo-spatial WM abilities predicted response to anomia treatment in their sample of individuals with aphasia. Perhaps the most important recent advancement is the growing evidence that STM/WM impairments are amenable to treatment, with a few reports documenting transfer effects to core levels of linguistic processing such as spoken sentence processing following STM/WM training (e.g., Harris, Olson, \& Humphreys, 2014; Zakariás, 
Keresztes, Marton, \& Wartenburger, 2016). Admittedly, the evidence base is not yet strong. However, the important contribution of this novel trend in aphasia treatment moves the focus from repeated exposure of a predetermined closed set of linguistic stimuli, structures, and representations or from strategies to offset solely linguistic deficits, to a more dynamic paradigm in which the underlying process of STM/WM that supports linguistic processing and perhaps the learning process in treatment, also becomes an intervention consideration (e.g., utilizing linguistic treatment stimuli that vary within and across treatment sessions).

From a patient's point of view, there is growing awareness that memory deficits are not identified nor duly addressed in aphasia or more broadly stroke rehabilitation. For instance, in the UK Stroke Association Stroke Survivors' Needs Survey (McKevitt et al., 2011), approximately $43 \%$ of respondents reported memory problems. Furthermore, $59 \%$ of those who reported memory problems regarded getting help with such problems as an unmet long-term need. Likewise, in an earlier study, both left hemisphere stroke survivors and their close relatives reported memory problems as one the most common, persistent cognitive concerns (VisserKeizer, Meyboom-De Jon, Deelman, Berg, \& Gerritsen, 2002). Whereas neither investigation distinguished the nature of these reported memory problems in relation to STM/WM, the crucial function of STM/WM as a gatekeeper of higher order cognition, and, consequently, every day functioning, does point towards serious consideration of STM/WM in clinical decision-making. This point is underscored by Sulleman and Kim (2015) who contended that the WM deficits in people with aphasia may undermine their ability to make cogent choices pertaining to aspects of their rehabilitation.

The inclusion of STM/WM subtests in clinically focused aphasia assessment batteries has existed for some time. For example, the ubiquitous digit span task, a STM test, was part of the 
Minnesota Test for Differential Diagnosis for Aphasia (Schuell, 1965), and it as well as other span tasks have continued to be found in current, highly popular tests (e.g., Psycholinguistic Assessments of Language Processing in Aphasia, Kay, Lesser, \& Coltheart, 1992;

Comprehensive Aphasia Test, Swinburn, Porter, \& Howard, 2005). The persistent inclusion of such memory tasks could be an indicator of the value test authors, and possibly clinicians, place on STM/WM assessment in clinical contexts. Nevertheless, a recent systematic review of the use of STM/WM tests in aphasia research highlighted several limitations and concerns (Murray, Salis, Martin, \& Dralle, 2016). The findings of the review revealed that a very limited number of standardized STM/WM tests, in the verbal and non-verbal domains, had robust psychometric properties. Common issues across tests included small standardization samples to elicit normative data, and poor validity as well as reliability properties ${ }^{1}$. Importantly, Murray and colleagues concluded that practice guidelines could not yet be forwarded as their review yielded no gold standard for evaluating STM/WM abilities in individuals with aphasia.

Accordingly, clinicians with an interest in aphasia management are faced with a quandary given such quality issues with currently available STM/WM tests, in the context of an evergrowing research base espousing the value of documenting the integrity of these memory abilities in individuals with aphasia. In particular, there has been no empirical investigation of how clinicians approach the assessment of STM/WM abilities when providing services to individuals with aphasia; that is, little is known about current clinical assessment practices (e.g., type and range of tests), opinions, or attitudes towards the assessment of STM/WM in aphasia. Whereas there has been some related research on assessment practices in other clinical

\footnotetext{
${ }^{1}$ Validity refers to several psychometric properties (e.g., construct, concurrent) that assess the extent to which a test actually measures what it purports to measure; reliability properties (e.g., test-retest, interrater) relate to stability of scores across time or assessors/raters (for a more detailed description, see Murray \& Clark, 2015).
} 
populations such as the management of cognitive-communication disorders in traumatic brain injury (Duff, Proctor, \& Haley, 2002; Frith, Togher, Ferguson, Levick, \& Dockin, 2014), and the assessment of communication impairments in stroke (Guo, Togher, \& Power, 2014; Vogel, Maruff, \& Morgan, 2010), to date no study has sought to identify clinical practices in terms of $\mathrm{STM} / \mathrm{WM}$ assessment in aphasia.

Consequently, the purpose of the current investigation was to survey speech-language pathology professionals, both clinicians and researchers, involved in aphasia management to describe the current state of STM/WM assessment practices. The specific aims of the survey were to: (1) investigate both views toward STM/WM assessment in individuals with aphasia as well as the types and frequency of STM/WM tests used with individuals with aphasia; and, (2) explore factors including educational background or occupation (e.g., researcher vs. clinician) and geographic location that may influence STM/WM assessment practices using inferential statistical methods. With some exceptions (cf., Frith et al., 2014), the exploration of such factors using inferential statistics is not a feature of surveys about practices of clinicians in speechlanguage pathology (Duff et al., 2002; Guo et al., 2014; Vogel et al., 2010). Frith and colleagues (2014) found, however, that the experience of clinicians working with individuals who had sustained traumatic brain injuries influenced assessment practices in several areas of communication. The authors also reported that clinicians in the USA or Canada routinely assessed some cognitive skills (e.g., problem-solving) more frequently than clinicians in the UK, Australia, or New Zealand. Given such findings for traumatic brain injury assessment practices, we were interested in determining if factors related to work experience such as occupation (i.e., those working in clinical vs. research settings) similarly influence STM/WM assessment practices in aphasia. For example, research settings typically offer greater support and more 
frequent opportunities to access the empirical literature base compared to clinical settings (Morris, Wooding, \& Grant, 2011; Rappolt \& Tassone, 2002). Therefore, it is plausible that those working in research versus clinical settings might have greater familiarity with and/or access to the evidence base describing the role of and assessment tools for STM/WM abilities in aphasia, which in turn could affect their use of and preference for certain STM/WM assessment tools. Likewise, the geographic factor identified by Frith et al. (2014) was of interest in the current survey study given that countries often differ in terms of their educational requirements for speech-language pathology professionals as well as their health care policy and thus access to speech-language pathology services for aphasia management. Indeed, prior research has determined geographic differences in aphasia management practices, albeit these have primarily focused on intervention rather than assessment issues (Code \& Petheram, 2011; Katz et al., 2000). With reference to the two countries (i.e., USA, UK) that became one of the foci of the analyses in the current study, there are differences in terms of education of speech-language pathology (SLP) clinicians as well as healthcare provision. For example, the required (preregistration with a professional body) degree for entry to practice SLP in the UK is either the Bachelor (three or four years, full-time) or Masters level (two years full-time), whereas in the USA it is the Masters level. The UK has a government-funded healthcare system, which employs the majority of SLP, whereas in the USA the healthcare system is for the most part private, with only limited government-funded healthcare services and SLP positions (e.g., Veteran's Administration healthcare facilities). Understanding whether such geographic and educational/occupational factors influence assessment practices could be relevant to informing clinical issues such as: (1) provision of mentorship or other forms of support to newly qualified SLPs; (2) service level workforce planning (e.g., is it necessary to recruit SLPs with higher 
educational qualifications to aphasia specialties?); (3) specifying guidelines for continuing professional development; and, (4) implications of relocation of SLPs between countries and also within services and clinical areas of specialty. In summary, the purpose of the survey is directly rooted to issues of clinical practice, with further specification of clinical implications towards the end of the paper.

\section{Method}

\section{Survey Content and Procedures}

The survey content was divided into five sections (see Appendix A) and took approximately 10-15 minutes to complete. The items comprising the survey content followed a forced-choice format as well as multiple-choice options, Likert scales, ranking of responses, and some optional sections for written open-ended responses from respondents. The items' content and organization within the survey were developed iteratively. In the first iteration, the third and second authors composed a set of items and designed the order of these items within the survey. The first author who suggested revisions in the order of items and survey sections as well as the wording of the items then reviewed this initial draft. Once this internal process was completed, two external reviewers also commented. One of the reviewers was based at an academic institution in the UK and had extensive experience in aphasia rehabilitation and healthcare research. The other reviewer was also based in the UK in a clinical setting; this reviewer also had extensive clinical and research experience in aphasia. Lastly, a doctoral student with research experience in survey development and clinical experience as a licensed SLP and two clinical supervisors at the second and third authors' educational institution trialed the survey to provide additional feedback about the items, survey design, and ease of survey completion. 
Section 1 aimed to elicit information about respondents' demographic backgrounds, clinical experience working with people with aphasia, type of clinical setting, and country of practice. Sections 2 and 3 elicited information about the STM/WM assessment practices of the respondents in their clinical setting, including the frequency with which they evaluated STM/WM as part of an aphasia assessment and their opinions about specific purposes of STM/WM in the clinical management of aphasia. Section 4 focused on the frequency of use of contemporary STM/WM tests and test batteries (both verbal and non-verbal). The choice of tests included in the survey was based partly on the findings of a recent systematic review of STM/WM test in aphasia (Murray et al., 2016) as well as the clinical and research experience of the authors (see Appendix D for a list of these tests). Additionally, there were two options for respondents to include self-rating questionnaires of functional memory abilities, and ratings of how often these were used. There were another three options for respondents to list other tests that had not been included in the survey questions and provide a frequency rating for such other tests. Finally, section 5 was concerned with ranking the most preferred tests (choice of three) from those listed in Section 4 of the survey. The survey ended with the option of adding any other comment the participant wanted to disclose.

The content of the survey was inputted on the website Qualtrics, a commercially available software designed for online surveys. The survey could be completed either on a computer or a smartphone and was open for completion from May to December of 2015. Ethical approval for the study was obtained from Indiana University Internal Review Board.

\section{Participant Recruitment}


A standard invitation e-mail message was created, inviting potential respondents and explaining the purpose of the study. The e-mail also provided definitions of STM/WM as well as the web link for accessing the survey and the expected time commitment. This invitation e-mail was distributed through the following e-mail lists and publications: American Speech and Hearing Association's (ASHA) Special Interest Group of Neurogenic Communication Disorders, ASHA's Clinicians and Researchers Collaborating (CLARC) Discussion Digest, the Royal College of Speech and Language Therapists (RCSLT), the Irish Association of Speech and Language Therapists, the Academy of Aphasia, the World Federation for NeuroRehabilitation, the British Aphasiology Society, and the Academy of Neurologic Communication Disorders and Sciences. The e-mail was also distributed through the National Aphasia Association newsletter, and the research newsletter of the RCSLT. A translation of the invitation e-mail from English to German was also carried out and disseminated through professional contacts of the authors in Germany and Switzerland.

\section{Data Analyses}

To address the first aim of the study, descriptive statistics for each completed quantitative question, which were automatically provided by Qualtrics, were reviewed. Additional reports were created to filter the responses for the write-in questions and recorded in Microsoft Excel. A bottom-up thematic analysis (Boyatzis, 1998) was completed for items that were open-ended and for which respondents provided comments. The first author (CS) reviewed all comments and categorized them into themes. Then, the second author (LM) also reviewed the comments and verified the previously identified themes. The few discrepancies that occurred were resolved by discussion. 
To address the second aim of the study, two binomial logistic regressions were carried out. Binomial logistic regression aims to predict the probability that a participant falls into one of two categories from the values of several explanatory variables (Agresti, 2007; Tabachnik \& Fidell, 2014). In our case, the categories were clinicians vs. researchers/clinicians (first regression) to examine education/occupation background, and participants from the USA vs. $\mathrm{UK}^{2}$ (second regression) to examine geographic location. With reference to these categories, we sought to examine how accurate this classification was when a number of additional variables were taken into account (i.e., responses to questions about assessment practices, experience, education) (cf., Agresti, 2007; Tabachnik \& Fidell, 2014). The regressions focused only on a subset of questions because those questions $(8,12-16)$ involved Likert ratings and were central to the purpose of the survey to reveal assessment practices.

Mindful of the relatively small sample size and that the robustness of regressions is influenced by the number of variables (Morgan, Leech, Gloeckner, \& Barrett, 2010), we used principal components analysis (PCA) to reduce the number of variables. We only applied PCA to questions 12 to 16 (five variables). We excluded question 8 (frequency of STM/WM assessment) because that question was conceptually different from questions 12 to 16 as it elicited information about perceived frequency of STM/WM assessment; in contrast, questions 12 to 16 focused on perceptions of the value of STM/WM assessment as part of aphasia management. Prior to the PCA being carried out, all variables showed at least one correlation of .3 (Kinnear \& Gray, 2008) (see Appendix B; Pearson correlations; all correlations are significant, $p \leq .004$, onetailed). We should note that the data entered into the PCA was not normally distributed which may minimize the reliability of results (Tabachnik \& Fidell, 2014). The PCA (which included a

\footnotetext{
${ }^{2}$ Other countries were not included because of the low numbers of respondents from those countries.
} 
varimax rotation) yielded two Factors (Eigen values $>1$ ). The two Factors combined accounted for $74 \%$ of the total variance. Before rotation, the initial communalities in the five variables ranged from .698 to .788 , suggesting sample size adequacy (Morgan et al., 2010). The rotated Factor pattern and final communality estimates are shown in Appendix $\mathrm{C}\left(\mathrm{h}^{2}=\right.$ communality estimates). The Factors reflected the question groupings into 'I believe' and 'I would' statements. To retain the meaningfulness of the reduced variables we termed Factor 1 'current attitudes towards STM/WM testing' reflecting questions starting with 'I believe' (Appendix A). Factor 2 was termed 'hypothetical attitudes towards STM/WM testing' reflecting questions starting with 'I would' (Appendices A and C). Finally, the two Factors were recoded as two variables (Bartlett method; Keho, 2012), which were used in the two regressions.

Returning to the binomial logistic regressions, the first analysis examined whether respondents who were solely clinicians vs. those with a dual researcher/clinician role had different assessment practices, taking into account educational qualification and experience. We operationalized assessment practices as the views expressed in response to the frequency of STM/WM test use (question 8) and other views expressed in questions 12 to 16 (Factors 1 and 2). In this model, the binomial response variable was clinician $(\mathrm{n}=95)$ and researcher/clinician ( $\mathrm{n}=50$; this number includes eight respondents who identified themselves solely as researchers). The few respondents ( $\mathrm{n}=7$, see Results) who had previously identified themselves as having another job description ("other") were classified as clinicians or researchers/clinicians based on how they characterized themselves in the written-in response to that part of the survey (e.g., one participant identified himself or herself as a "student clinician" and another identified himself or herself as an "academic clinical supervisor"); based on the written-in responses, all these participants were classified as clinicians. In addition to assessment practices (i.e., question 8, 
Factors 1, and 2), two further variables were considered: (1) years of experience working with people with aphasia; and (2) level of education (respondents with Ph.D. or Clinical Doctorate $[n$ $=50]$, and respondents with a Bachelors, Masters or other qualifications $[n=95]$ ).

The second regression examined whether respondents who were located in the USA $(n=$ $67)$ and those in the UK $(n=29)$ had different assessment practices (i.e., question 8 , Factors 1 , and 2), taking into account, level of education, experience, and whether they had solely a clinician role or a dual researcher/clinician role. In addition to assessment practices (question 8, Factors 1, and 2), three further variables were considered: (1) years of experience in working with people with aphasia; (2) level of education (respondents with Ph.D. or Clinical Doctorate $[n$ $=29$ ], and respondents with a Bachelors or Masters $[n=67])$; and (3) clinicians $(n=69)$ and researchers/clinicians $(n=27)$.

\section{Results and Discussion}

A total of 302 individuals responded to the survey, but 157 of these individuals failed to finish responding to all required items. Therefore, the total number of respondents who completed the survey was 145 . Reporting of the results and discussion adheres to the structure of the survey, followed by findings from the regression analyses.

\section{Survey Section 1: Demographic Information Characteristics}

About half of the respondents (48\% of 145$)$ were located in the USA, $21 \%$ in the UK, and the remaining $31 \%$ in other countries from around the world. More specifically, $6 \%$ of the respondents were located in Germany, 4\% in Switzerland, and 3\% in Denmark. The remaining were located in Australia, Austria, Benin, Canada, Chile, Cyprus, France, Greece, India, Ireland, 
Italy, Montenegro, New Zealand, Norway, Portugal, Singapore, South Africa and South Korea; in these countries, the number of respondents completing the survey ranged from one to three.

The majority ( $63 \%$ of 145$)$ identified themselves as SLP clinicians, whereas $26 \%$ were both a clinician and a researcher, $6 \%$ were researchers, and $5 \%$ responded as having another job description (e.g., student clinician, academic clinical supervisor). In terms of educational background, most respondents (69\%) had Bachelors, Masters, or other qualifications, and 31\% had a Doctorate (either Ph.D. or Clinical Doctorate).

In terms of years of clinical experience in working with people with aphasia, $38 \%$ of the sample had 1 to 10 years, $35 \%$ had 11 to 20 years, and $27 \%$ had 21 or more years of experience. The clinical settings at which respondents practiced were as follows: $36 \%$ in inpatient settings, $33 \%$ in mixed settings, and $32 \%$ in outpatient community rehabilitation or private practice. With respect to the respondents' estimates of the number of people with aphasia they assessed per year, $41 \%$ assessed 1 to 20 people, $35 \%$ assessed 21 to 40 people, and $23 \%$ assessed 41 or more people. The ensuing results and discussion

\section{Survey Section 2: Mechanics of STM/WM Testing}

This section (and Section 3 below) sought to understand the views of clinicians toward STM/WM assessment in aphasia. In terms of relative frequency of assessing STM/WM (question 8), $60 \%$ of the 145 respondents reported that they regularly assessed STM/WM (i.e., "every time" $=30 \%$, or "often" $=30 \%$ ) as part of providing services to people with aphasia. Proportions of responses from the remaining respondents were as follows: "sometimes" (26\%), "seldom" (11\%), or "never" (3\%).

Respondents who did not assess STM/WM "every time" or "often" were asked if another 
professional in their team carried out STM/WM testing of individuals with aphasia (question 9).

Of the 58 respondents who fell into this group, $21 \%$ reported that a psychologist was carrying out STM/WM testing, and 17\% reported that STM/WM testing was completed by an occupational therapist (question 10). The remaining $62 \%$ of these 58 respondents responded that no one else was carrying out STM/WM testing of individuals with aphasia. These respondents were also asked a follow-up question (question 11) that offered response choices reflecting why STM/WM testing was not carried out at their clinical location. Results for this question were as follows, keeping in mind that respondents were asked to select all responses choices that applied to their situation (i.e., percentages across responses exceed 100\%): Available STM/WM tests were outdated (3\%), a lack of training or experience with cognitive tests (11\%), lack of time (17\%), access to tests (17\%), available tests not suitable for those with aphasia $(53 \%)$, and "other" (50\%). For the "other" response choice, respondents could write-in additional reasons for not assessing STM/WM memory; an analysis of their written-in responses is provided next.

\section{Content Analysis of Respondents' Comments}

Respondents who indicated that they did not assess STM/WM at all (five of the 145) when providing aphasia management were asked to provide reasons, and the following response themes emerged from a content analysis of their written-in responses (associated number of comments for each category in brackets): "STM/WM was not a primary area of concern" ( $\mathrm{n}=8)$, "STM/WM was assessed but with informal assessment procedures only" ( $\mathrm{n}=4)$, "language ability of clients was too low for STM/WM assessment" (n=4), "lack of funding for obtaining formal STM/WM assessments" ( $\mathrm{n}=1)$, "STM/WM testing was only completed if needed" $(\mathrm{n}=1)$. 


\section{Survey Section 3: Views towards STM/WM Testing}

Respondents completed three ${ }^{3}$ questions (questions 12, 13, 14, five-point Likert scale). Two additional questions $(15,16)$ focused on accessibility of STM/WM tests and the evidencebase about STM/WM in aphasia management. Results from this section of the survey are summarized in Table 1.

Whereas overall the findings indicated that the majority of respondents supported the value of and reported regularly including STM/WM assessment as part of aphasia management, several clinical practice issues were identified. The vast majority of respondents (e.g., $84 \%$ of 145 respondents) strongly agreed or agreed that STM/WM assessment results have value when making decisions about aphasia assessment and treatment selection as well as progress and prognosis; however, a smaller proportion, albeit still a majority, reported regularly assessing the STM/WM abilities of their clients with aphasia (i.e., $60 \%$ reported including STM/WM testing "every time" or "often"). Furthermore, among respondents who reported not regularly assessing the STM/WM abilities of their clients with aphasia, the majority (62\%) responded that no one else at their clinical setting was doing so either for this patient population. These findings highlight a gap between perceptions of value versus actual practices.

Several factors may underlie this mismatch between the perceived value of STM/WM assessment, which has empirical support in the aphasia literature (e.g., Martin \& Ayala, 2004; Martin \& Allen, 2008), and reported clinical practices. The most frequently cited reason (53\%) was that available tests were not considered suitable for people with aphasia. Although it is unclear from the findings of the survey as to the reason why respondents did not consider available STM/WM tests suitable, possible reasons from published literature suggest the

\footnotetext{
${ }^{3}$ We should acknowledge that in this section there was an accidental repetition of the question about documenting treatment progress. We only included data from the first occurrence of the question.
} 
following: (1) number processing skills in the digit span task (a popular STM measure) is often impaired in aphasia and may therefore provide a less than accurate measure of STM/WM (DeDe, Ricca, Knilans, \& Trubl, 2014); (2) many STM tests have spoken repetition response demands and thus, severity of the aphasic repetition deficit will impact performance of such tests (Howard \& Franklin, 1990); and, (3) lexical retrieval deficits may interfere with verbal STM/WM tests (Warrington \& Shallice, 1969). The suitability of STM/WM tests for people with aphasia may also relate to aphasia severity, which was also noted by Murray et al. (2016) who found that persons with severe/global aphasia were under-represented in research of STM/WM in aphasia. Other less frequently cited reasons were limited access to STM/WM tests (17\%) and lack of training or experience with cognitive tests $(11 \%)$. Lack of time was also reported $(17 \%)$, presumably for more comprehensive assessment of a person's abilities that includes STM/WM functioning in addition to other cognitive-linguistic abilities and psychosocial functioning; Vogel et al. (2010) similarly identified time constraint issues in their survey of communicative assessment practices for stroke survivors in the acute stages of recovery.

Survey Section 4: Frequency of Use of Specific STM/WM Tests

This section of the survey asked about how frequently clinicians used specific tests in their clinical practice in order to relate this to psychometric quality markers of STM/WM tests. Therefore, this section required respondents to indicate how frequently they used the specific formal STM/WM tests and subtests listed in Appendix D. Table 2 summarizes their rankings in terms of popularity and availability. Combining the proportion of respondents who chose the rating categories of "always" or "sometimes" derived the rankings in the most popular column. The three most popular tests, Cognitive Linguistic Quick Test (CLQT), Comprehensive Aphasia 
Test (CAT), and Token Test, were appraised by Murray et al. (2016). The Design Memory subtest of CLQT was found to have excellent construct validity and fair content/face validity, but in other aspects of validity (e.g., predictive, discriminant) and reliability (e.g., test-retest), it was weak. The Digit Span of the CAT was reported to have excellent construct validity, and predictive and discriminant aspects of validity were present. In terms of reliability, it had fair test-retest reliability and inter-rater reliability but other psychometric aspects (e.g., concurrent validity, measurement error) were poor. Finally, the Token Test only fared well in terms of interrater reliability but was poor in all other aspects of reliability and validity.

The rankings in the least popular column were derived from the proportion of respondents who chose the rating category "never." The tests most often reported as "not available" were the Wide Range Assessment of Memory and Learning (WRAML), the Scales of Cognitive and Communicative Ability for Neurorehabilitation (SCCAN), and Test of Memory and Learning (TOMAL). Similarly, these tests were not being used by aphasia researchers in the literature reviewed by Murray et al. (2016); in the case of the SCCAN and TOMAL (particularly the version for older adults), Murray and colleagues hypothesized that their absence among the studies included in the systematic review may have related to these tests' relatively recent publication dates. Respondents also had the opportunity to write in the names (see Appendix E) and rank their frequency of use of any other STM/WM tests that they used but were not listed in the survey. All of these "other" tests were ranked as being used either "always" or "sometimes" by the respondents who listed them.

In this section of the survey respondents were also asked about self-rating questionnaires or scales as a means to assess clients' subjective perceptions of their own functional memory abilities. Many respondents ( $49 \%$ of 145 respondents) reported that they never used self-rating 
questionnaires, while $35 \%$ reported using them only with those clients who had adequate reading/listening skills (question 17 ). Fewer respondents (8\%) reported that they used these questionnaires in every aphasia assessment, and $2 \%$ reported using them only with those clients who had a caregiver who could complete the questionnaire as a proxy. The remaining $6 \%$ of the respondents wrote in the following responses regarding their use of self-rating questionnaires: use informal vs. formal self-rating questionnaires $(n=2)$, only use self-rating questionnaires if requested or recommended $(n=3)$, infrequent use of self-rating questionnaires $(n=1)$, and unable to use because such questionnaires are not available in his/her language $(n=1)$. One additional written-in response indicated that the participant viewed self-rating questionnaires more appropriate and only empirically substantiated for obtaining information on quality of life versus memory impairments. Respondents were also asked to write in the names of the self-rating questionnaires that they used as well as rate their frequency of use of these questionnaires. Each of the questionnaires listed in Appendix F was rated as being used "always" or "sometimes." Not listed in Appendix $\mathrm{F}$ is the response of one participant who commented that $\mathrm{s} /$ he could not recall the name of the questionnaire, which s/he "sometimes" used. We should note that not all of the questionnaires in Appendix F that respondents reported using tap into functional memory abilities and the implication of STM/WM problems for activities in daily living (e.g., Token Test; repetition of sentences).

Use of questionnaires to document persons' perceptions of their own memory abilities was relatively low. That is, $49 \%$ of respondents reported never using these types of measures. In reference to the International Classification of Functioning (World Health Organisation, 2001), which is widely used in rehabilitation research to understand not only impairments themselves but also their implications for the person and his or her participation in daily activities and social 
roles, standard STM/WM tests focus on 'body structures and functions,' whereas self-rating questionnaires of memory focus on the 'activities and participation' domain. Although strictly speaking memory self-rating questionnaires do not focus solely or explicitly on STM/WM but also related cognitive abilities, limitations of STM/WM can interfere with activities of daily living that involve communication (e.g., Vallat-Azouvi, Pradat-Diehl, \& Azouvi, 2012). The inclusion of such questionnaires in the aphasia assessment process can thus provide a more holistic view of a person's STM/WM and related cognitive abilities and how difficulties in such cognitive areas may interfere with activities of daily living. Such questionnaires could also enable identifying individuals who need on-going referral to other professionals and services (e.g., occupational therapy, clinical psychology), and thus address the concerns that McKevitt et al. (2011) revealed in terms of adequate service provision for stroke survivors with memory problems. Indeed, about a third of respondents (35\%) reported that they only used such questionnaires with those people who had adequate reading/listening skills, and presumably milder forms of aphasia.

\section{Survey Section 5: Preference of Assessment Tools}

This section sought to distinguish between frequency of use of actual tests (cf., Section 4) versus preference of specific tests and types of tests in terms of modality (e.g., auditory-verbal, visuo-spatial). Although the two sections (and concepts of frequency of use vs. preference) are related, they are not identical. Therefore, this final section of the survey prompted respondents to rank their top three preferred STM/WM tests. Via a drop-down menu, they were given all of the tests in Appendix D as well as the following: "other test," "self-rating questionnaire," and "no preference." The CLQT (19\% of 145 respondents) was chosen most often as the most preferred 
test, followed by the CAT (14\%), and "no preference" (11\%). The tests that were least frequently chosen as a preferred test were the TOMAL (1\%), WRAML (2\%), and the Rey-Osterrieth Complex Figure (ROCF) (4\%).

The respondents were also asked to rank their preference of STM/WM test type (i.e., not a specific commercially available test/subtest but rather STM/WM task like digit or pointing span) by a "drag and drop" cursor function to express their choices (i.e., recall, semantic memory, digit span, tapping span, list learning, other) from "(1) Most Preferred" to "(6) Least Preferred." To identify a clearer pattern among the various preferences reported by the respondents, the six original preference choices were combined into three broader categories: “Most Preferred (1-2)," "Moderately Preferred (3-4)," and "Less Preferred (5-6)." Recall (52\%) was the most preferred test type overall, followed by Digit Span (46\%) and Semantic Memory (43\%). The least preferred test type that was most frequency cited was "other, please specify" (37\%); however, this could be due to some respondents not having another test in mind and thus choosing it via a process of elimination. After "other," the least preferred subtest types were Tapping Span (26\%) and List Learning (14\%).

In terms of preferences of assessment tools (question 18), the most popular was the CLQT, followed by the CAT, and lastly the Token Test. It could be that the comprehensive designs of the CLQT and CAT in terms of assessing a range of core linguistic and cognitive abilities make them particularly appealing for clinical use. Similarly, the Token Test can provide information about not only spoken sentence comprehension skills, but also if these are negatively influenced by STM demands (Salis et al., 2015). Additionally, the Token Test is part of the Aachen Aphasia Test, which is a popular aphasia battery in German-speaking countries and has been adapted in other European languages. It should be born in mind that despite the relative 
popularity of these tests, their psychometric properties have been questioned (Murray et al., 2016), so caution should be exercised in interpreting their results. For example, Murray et al. found that the CLQT design memory subtest, despite excellent construct validity and fair content/face validity, was poor in terms of reliability, concurrent validity, and measurement error and lacked information regarding its predictive or discriminant validity. The least popular tests in the current survey were the Corsi blocks and tapping span, which assess visuo-spatial STM. Their lack of popularity may reflect respondents' priorities in assessing verbal STM/WM, rather than visuo-spatial STM in persons with aphasia. Furthermore, only recently the prognostic potential of non-verbal STM/WM skills has begun to be investigated more systematically (Harnish \& Lundine, 2015). The Wechsler Memory Scale (WMS) was also unpopular, even though digit span, one of its subtests, has been identified as the most frequently used measure of STM/WM functioning in aphasia research (Murray et al., 2016). The WMS may lack popularity because it contains many subtests that extend beyond STM/WM, and clinicians can only purchase the WMS if they have a graduate degree in psychology. Finally, three tests (WRAML, SCCAN, TOMAL) were frequently cited as unavailable. Interestingly, these are relatively recently published tests, and consequently may not as yet have become popular in clinical practice.

\section{Final Survey Question: Additional Comments}

This section enabled us to investigate issues that respondents themselves wished to mention or discuss. Thirty-five of the 145 respondents provided written-in responses in this section of the survey (question 21). The analysis revealed the following themes. The numbers in 
brackets refer to the number of times comments or parts of comments (in the case of lengthy responses) that fell within that thematic category. Examples are verbatim.

(a) test preference $(\mathrm{n}=11)$ example: "I like the De Renzi \& Nichelli (1975) test because it has norms for left and right brain damage with and without aphasia"

(b) appropriateness of STM/WM tests for persons with aphasia $(\mathrm{n}=7)$ example: "I do not feel that you can truly measure working memory as a cognitive function when you have someone with aphasia"

(c) STM/WM other profession's remit ( $\mathrm{n}=6)$ example: "our OT uses RBANS [Repeatable Battery of the Assessment of Neuropsychological Status]"

(d) limited knowledge of tests featuring in the survey $(n=6)$ example: "I have not heard of most of these assessments"

(e) limitations imposed by clinical setting $(\mathrm{n}=5)$ example: "in the acute hospital phase there is not always time for this" (STM/WM assessment)

(f) comments about the content of the survey $(n=4)$ example: "the last few questions of the survey were not relevant to me because I do not assess STM/WM"

(g) test unavailability in clients' language $(\mathrm{n}=4)$ example: "these tests do not exist in German"

(h) comments about the design of the survey $(n=3)$ 
example: "I dislike opting for never - there would be occasional people where I might use a verbal test if the aphasia was rapidly resolving or I was confident the test was still valid. But there is no 'rarely' option!"

(i) value of STM/WM assessment $(\mathrm{n}=1)$ example: "this is an area of need for certain"

(j) limitation in accessing STM/WM tests $(\mathrm{n}=1)$ example: "I'm not allowed to purchase additional tests due to funding" These comments provided some further insights that underscored views about STM/WM assessment that featured in other parts of the survey as negative and positive themes. Most comments disclosed preference of particular STM/WM tests and this was the predominant theme featuring in this question. Beyond that, the appropriateness of STM/WM for persons with aphasia was also highlighted as a concern, followed by the idea that assessment of STM/WM as not being an SLP's priority or responsibility. Another reason for not assessing STM/WM may be uncertainty about possible treatment. This raises the issue that interdisciplinary work with neuropsychologists may be necessary to be able to know what to do with STM/WM assessment results in relation to choice of related treatment. This is evident in the theme ' $\mathrm{STM} / \mathrm{WM}$ other professional's remit.' Limited knowledge of tests came primarily from unavailability of tests in the respondents', and consequently the clients' own language, a point that reflects the inevitable bias of the survey on English-based tests. Limitations imposed by clinical setting were another theme, which can be construed as a barrier in implementing STM/WM assessment. Also, respondents expressed helpful comments about the design of the survey and one saw value in STM/WM assessment. 


\section{Results and Discussion from the Regression Analyses}

The second aim of the survey was to explore factors including occupation (e.g., researchers vs. researchers/clinicians) and geographic location that may influence STM/WM assessment practices as part of aphasia management. These analyses were motivated from the relative lack of knowledge in the literature as to how specific clinician-related factors may affect perceptions and practices as well as the possible influence of different educational and healthcare systems on assessment practices.

The first regression analysis sought to understand whether a clinical or dual role (researcher/clinician) influences attitudes towards STM/WM assessment practices. The analysis (see Table 3), which sought to predict response differences between clinicians and researchers/clinicians, was statistically significant, $\chi^{2}(5)=60.934, p<.001$. This model had $82.5 \%$ group classification accuracy. Nagelkerke's $R^{2}$ of .481 indicated a moderate relationship between prediction and classification. The Hosmer and Lemeshow test was not statistically significant, $\chi^{2}(8)=2.726, p=.95$, suggesting a good fit in terms of sampling adequacy. The Wald $\chi^{2}$ tests showed that: Factor 1 (questions 12, 13, 14), and educational qualification made significant contributions to the model, with education showing a greater effect than Factor 1. Question 8 (frequency of STM/WM assessment) did not make a significant contribution.

The variables that made significant contributions to the model were primarily educational qualification, followed by Factor 1 (i.e., 'current attitudes towards STM/WM testing'). As the majority of clinicians did not have Ph.D. degrees, Factor 1 significantly predicted clinician or dual role. The negative beta value (educational qualification) suggests that researchers/clinicians rated 'I believe' questions more highly, possibly because of greater knowledge about STM/WM. Frequency of STM/WM assessment did not predict whether or not someone was a clinician, or a 
researcher/clinician. Experience in working with people with aphasia was not a significant variable either. Factor 2 (i.e., 'hypothetical attitudes towards STM/WM testing') did not make a significant contribution to the model, suggesting that both clinicians and researchers/clinicians appeared to have similar perceptions about STM/WM assessment in aphasia, and, indirectly, the current status of the evidence base regarding STM/WM abilities in aphasia. In summary, there was similarity between the assessment practices of clinicians and researchers.

The second regression examined whether assessment practices differed between respondents in the USA and UK. This model involved the same variables as the first regression, and also, considered occupation (i.e., the clinician vs. researcher/clinician distinction). This regression (see Table 4) was not statistically significant, $\chi^{2}(6)=9.606, p=.142$. This model had $79.2 \%$ group classification accuracy, and Nagelkerke's $R^{2}$ of .135 indicated a weak relationship between prediction and classification. The Hosmer and Lemeshow test was not statistically significant, $\chi^{2}(8)=12.046, p=.149$, suggesting a good fit in terms of sampling adequacy. The Wald $\chi^{2}$ tests showed that only question 8 (frequency of STM/WM assessment) made a significant contribution to the model. None of the other variables were significant.

The second regression suggests that views and attitudes towards STM/WM assessment in USA and the UK were similar. Educational qualification, occupation, and level of experience did not significantly contribute to the model. Neither factor (i.e., 'current attitudes towards STM/WM testing,' 'hypothetical attitudes towards STM/WM testing') was significant either. The only variable that suggested a statistical difference between USA and UK respondents was the frequency with which respondents in the USA and UK assess STM/WM. A post-hoc descriptive analysis of ratings showed that the modal rating for this question was 5 (mean=3.9) for USA respondents and 3 (mean=3.3) for UK respondents. This suggests that compared to SLP 
professionals in the UK, SLP professionals in the USA may be likely to assess STM/WM more frequently. However, the second regression as a whole indicated that assessment practices and views towards STM/WM testing in aphasia management are more similar than different in the USA and UK.

\section{Clinical Implications}

Several clinical implications arise from the survey findings. First, although the majority of respondents reported assessing STM/WM as part of aphasia management services, the choice of tests in terms of popularity ratings suggested that clinicians should scrutinize the psychometric properties of the tests, and if weak, interpret the results cautiously and/or be judicious in their use. For example, tests with poor discriminant validity may not be sensitive enough to detect presence of a STM/WM impairment; in such cases, if feasible, it would be useful to administer more than one test to assess STM/WM and also use information from other assessment methods such as interviewing the patient and/or carer(s), as well as other professionals who provide care for a particular patient regarding STM/WM concerns. A couple of written-in test choices were tests not developed for the assessment of STM/WM abilities (e.g., Thematic Apperception Test; Raven's Progressive Matrices). Some respondents were using STM/WM tests or subjective measures with unknown psychometric properties and/or without normative data such as the matching listening span subtest of the Psycholinguistic Assessments of Language Processing in Aphasia (PALPA; Kay et al., 1992), The Brain Injury Workbook Questionnaire (Powell, 2013), or self-created number lists. Clearly, preference should be given to tests with stronger psychometric properties, which ideally are based on contemporary normative data and stimuli. In their review, Murray and colleagues (2016) found that none of the tests that they appraised 
received high quality markers across different aspects of validity, reliability, and measurement error. Based on findings of that appraisal, and mindful that none of the tests included in that review was consistently of high quality across all relevant psychometric properties, the following $\mathrm{STM} / \mathrm{WM}$ tests could be recommended as being relatively better choices for clinical use than others. First, the digit span of the CAT (Swinburn et al., 2005) had excellent construct validity, and was judged to have predictive and discriminant validity as well as inter-rater reliability; similarly, other versions of the digit span were found to be of relative good quality (Wechsler, 1981, 2000). In terms of visuo-spatial tests, the visual tapping span version by Wechsler (1997) was of higher quality than other similar tests. Mindful of an inherent limitation in the 2016 review by Murray et al. (i.e., specificity of the inclusion criteria), if clinicians have at their disposal tests that were not included in that review, the test appraisal checklist used by Murray et al. could be adopted to appraise other tests. As a scientific community, and in concert with recommendations by Murray et al., more emphasis should be given to the establishment of a "gold standard" (cf., DeDe et al., 2014), not only for STM/WM testing but also more widely for language assessment in aphasia.

Second, for those respondents, albeit a small proportion (11\%), who expressed that a lack of training or experience with cognitive tests is a perceived barrier to use of STM/WM tests, relevant opportunities for continuing professional development, ideally tailored to the needs of speech-language pathologists who work with people with aphasia, should be offered.

Third, in settings where clinicians either have no access to STM/WM tests or assessment time is limited, inter-professional relationships should be fostered with psychology, occupational therapy, and/or other relevant healthcare team members that would enable sharing of relevant assessment findings as well as mutual discussion and interpretation of information elicited from 
STM/WM testing in relation to communication skills and rehabilitation goals. To broaden access to tests, clinicians could consider both obtaining test materials and norms from publications (e.g., Lehman \& Tompkins, 1998), or contacting authors of test materials that have been developed primarily for research purposes (e.g., DeDe et al., 2014; Mayer \& Murray, 2012), mindful of the need to critically appraise such tests (as discussed earlier). Another free resource that could be useful is the National Institutes of Health Toolbox Cognition Battery (Tulsky, Carlozzi, Chiaravallotti, Beaumont, Kisala, Mingas, Conway \& Gershon, 2014; also see, http://www.healthmeasures.net/explore-measurement-systems/nih-toolbox).

Fourth, there remains a need to expand current STM/WM assessment practices to include, when appropriate, self-rating questionnaires of memory functioning. In comparison to traditional STM/WM tests, such questionnaires offer insights into participation in social activities that involve communication and carry a heavy burden on STM/WM (Vallat-Azouvi et al., 2012).

Finally, the survey results also highlight issues that require additional attention in the aphasia empirical literature. For example, several respondents shared the rightly justified perception that many STM/WM tests are not suitable for people with aphasia. Such concerns warrant further systematic investigation that would delineate as far as possible the relative involvement of STM/WM impairments (in relation to spoken vs. visual language modalities as well as non-verbal aspects) to core linguistic domains. Relatedly, respondents' apprehension about the suitability of memory functioning self-rating questionnaires for people with aphasia raises the issue of designing and validating questionnaires that are 'aphasia friendly,' ideally with the involvement of people with aphasia themselves and their carers. 
Before drawing key conclusions from the survey findings, we wish to highlight some limitations of the study. Although the survey reached respondents from a relatively large range of countries, the number of respondents was relatively small. The lack of responses internationally limits the transnational reach of this project. It is therefore unclear how representative the views expressed by the respondents are of clinicians across countries. This limits the generalization of the findings from the analyses. Another limitation inherent in surveys is that the sample of respondents is not random. For example, it is likely that that the survey may have attracted respondents (clinicians and researchers) with a special interest in STM/WM and other cognitive abilities beyond language in aphasia. If this were the case, the survey did not reach respondents whose clinical practice is in aphasia but have other special interests (e.g., linguistic assessment, functional communication). Furthermore, to obtain a larger and broader participant sample, other channels could have been used to communicate with potential respondents (e.g., hard copy publications of newsletters).

In conclusion, the findings from this survey study showed support for STM/WM assessment in aphasia. Most respondents reported regular inclusion of STM/WM tests as part of aphasia management, with some variation in STM/WM assessment views and practices related to factors such as occupation (i.e., clinician vs. researcher/clinician); the findings were, however, similar for respondents practicing in the UK vs. USA. The survey results highlighted the importance clinicians (and researchers) place upon STM/WM testing in aphasia because of its perceived effectiveness in making prognostic decisions, selecting treatment procedures, and documenting treatment progress. However, our investigation also revealed issues in terms of accessibility of STM/WM tests for clinicians and limitations in the current evidence/knowledge base of STM/WM testing in aphasia. A need for enhanced translation of the current evidence 
base to clinical practice was identified given that not all respondents regularly assessed STM/WM abilities when providing services to individuals with aphasia, two of the most frequently used tests have inadequate psychometric properties, and few respondents reported regular use of memory self-perception scales or questionnaires. Finally, the survey highlighted avenues for further research that would help address this important yet relatively neglected domain of clinical enquiry and knowledge. 
Acknowledgements: We are grateful to all respondents who completed the survey and the organizations as well as persons who promoted the survey. We would also like to thank Pam Enderby and Fiona Stewart who provided feedback on the content of the survey. 


\section{References}

Agresti, A., (2007). An Introduction to Categorical Data Analysis. Hoboken, NJ: Wiley.

Baddeley, A. (2012). Working memory: Theories, models, and controversies. Annual Review of Psychology, 63, 1-29.

Baldo, J. V., Katseff, S., \& Dronkers, N. F. (2012). Brain regions underlying repetition and auditory-verbal short-term memory deficits in aphasia: Evidence from voxel-based lesion symptom mapping. Aphasiology, 26, 338-354.

Boyatzis, R. E., (1998). Transforming Qualitative Information Thematic Analysis and Code Development. Thousand Oaks, CA: Sage.

Cahana-Amitay, D., \& Albert, M. L. (2015). Redefining Recovery from Aphasia. New York: Oxford University Press.

Caplan, D., Michaud, J., \& Hufford, R. (2013). Short-term memory, working memory, and syntactic comprehension in aphasia. Cognitive Neuropsychology, 30, 77-109.

Code, C., \& Petheram, B. (2011). Delivering for aphasia. International Journal of Speech-Language Pathology, 13, 3-10.

Conway, A. R. A., Cowan, N., Bunting, M. F., Therriault, D. J., \& Minkoff, S. R. B. (2002). A latent variable analysis of working memory capacity, short-term memory capacity, processing speed, and general fluid intelligence. Intelligence, 30, 163-183.

DeDe, G., Ricca, M., Knilans, J., \& Trubl, B. (2014). Construct validity and reliability of working memory tasks for people with aphasia. Aphasiology, 28, 692-712.

Duff, M., Proctor, A., \& Haley, K. (2002). Mild traumatic brain injury (MTBI): Assessment and treatment procedures used by speech-language pathologists (SLPs). Brain Injury, 16, 773-787. 
Eling, P. (2015). Kurt Goldstein's test battery. Cortex, 63, 16-26.

Frith, M., Togher, L., Ferguson, A., Levick, W., \& Dockin, K. (2014). Assessment practices of speech-language pathologists for cognitive communication disorders following traumatic brain injury in adults: An international survey. Brain Injury, 28, 1657-1666.

Guo, Y. E., Togher, L., \& Power, E. (2014). Speech pathology services for people with aphasia: What is the current practice in Singapore. Disability and Rehabilitation, 36, 691-704.

Harnish, S. M., \& Lundine, J. (2015). Nonverbal working memory as a predictor of anomia treatment success. American Journal of Speech-Language Pathology, 24, S880-S894.

Harris, L., Olson, A., \& Humphreys, G. (2014). The link between STM and sentence comprehension: A neuropsychological rehabilitation study. Neuropsychological Rehabilitation, $24,678-720$.

Howard, D., \& Franklin, S. (1990). Memory without rehearsal. In G. Vallar \& T. Shallice (Eds.), Neuropsychological Impairments of Short-term Memory (pp. 287-318). Cambridge, UK: Cambridge University Press.

Katz, R., Hallowell, B., Code, C., Armstrong, E., Roberts, P., Pound, C., \& Katz, L. (2000). A multi-national comparison of aphasia management practices. International Journal of Language and Communication Disorders, 35, 303-314.

Keho, Y. (2012). The Basics of Linear Principal Components Analysis, Principal Component Analysis, P. Sanguansat (Ed.), ISBN: 978-953-51-0195-6, InTech, Available from: http://www.intechopen.com/books/principal-component-analysis/the-basics-of-principalcomponent-analysis

Kinnear, P. R., \& Gray, C. D. (2008). SPSS 15 Made Simple. Hove, UK: Psychology Press. 
Lang, C. J. G., \& Quitz, A. (2012). Verbal and nonverbal memory impairment in aphasia. Journal of Neurology, 259, 1655-1661.

Lehman, M. T., \& Tompkins, C. A. (1998) Reliability and validity of an auditory working memory measure: Data from elderly and right-hemisphere damaged adults. Aphasiology, 12, 771-785.

Martin, N., \& Ayala, J. (2004). Measurements of auditory-verbal STM span in aphasia: Effects of item, task, and lexical impairment. Brain and Language, 89, 464-483.

Martin, R. C., \& Allen, C. M. (2008). A disorder of executive function and its role in language processing. Seminars in Speech and Language, 29, 201-210.

Mayer, J. F., \& Murray, L. L. (2012). Measuring working memory deficits in aphasia. Journal of Communication Disorders, 45, 325-339.

McKevitt, C., Fudge, N., Redfern, J., Sheldenkar, A., Crichton, S., Rudd, A. ... Wolfe, C. D. A. (2011). Self-reported long-term needs after stroke. Stroke, 42, 1398-1403.

Morgan, G. A., Leech, N. L., Gloeckner, G. W., \& Barrett, K. C. (2010). IBM SPSS for Introductory Statistics. London: Taylor \& Francis.

Morris, Z. S., Wooding, S., \& Grant, J. (2011). The answer in 17 years, what is the question: Understanding time lags in translational research. Journal of the Royal Society of Medicine, 104, 510-520.

Murray, L. L. (2012). Direct and indirect treatment approaches for addressing short-term or working memory deficits in aphasia. Aphasiology, 26, 317-337.

Murray, L. L., \& Clark, H. M. (2015). Neurogenic Disorders of Language and Cognition: Evidence-based clinical practice. Austin, TX: Pro-Ed.

Murray, L. L., \& Mayer, J. (2016). Extra-linguistic cognitive considerations in aphasia 
management. In I. Papathanasiou, P. Coppens \& C. Potagas (Eds.), Aphasia and Related Neurogenic Communication Disorders, $2^{\text {nd }} E d$ (pp. 129-150). Sudbury, MA: Jones \& Bartlett.

Murray, L. L., Salis, C., Martin, N., \& Dralle, J. (2016). The use of standardised shortterm and working memory tests in aphasia research: A systematic review. Neuropsychological Rehabilitation, 1-43.

Powell, T. (2013). The Brain Injury Workbook: Exercises for cognitive rehabilitation $\left(2^{\text {nd }}\right.$ Ed.). Bicester, UK: Speechmark.

Rappolt, S., \& Tassone, M. (2002). How rehabilitation therapists gather, evaluate, and implement new knowledge. Journal of Continuing Education in the Health Professions, 22, 170180.

Rottschy, C., Langner, R., Dogan, I., Reetz, K., Laird, A. R. Schulz, J. B., Fox, P. T., \& Eickhoff, S. B. (2012). Modelling neural correlates of working memory: A coordinate-based meta-analysis. NeuroImage, 60, 830-846.

Salis, C., Kelly, H., \& Code, C., (2015). Assessment and treatment of short-term and working memory impairments in stroke aphasia: A practical tutorial. International Journal of Language and Communication Disorders, 50, 721-736.

Schuell, H. (1965). The Minnesota Test for Differential Diagnosis of Aphasia. Minneapolis: University of Minnesota Press.

Schuell, H., Jenkins, J. J., \& Jiménez-Pabón, E. (1964). Aphasia in Adults: Diagnosis, prognosis and treatment. London: Harper Row.

Sulleman, S., \& Kim, E. (2015). Decision-making, cognition, and aphasia: Developing a foundation for future discussions and inquiry. Aphasiology, 29, 1409-1425. 
Swinburn, K., Porter, G., \& Howard, D. (2005). The Comprehensive Aphasia Test. Hove, UK: Psychology Press.

Tabachnik, B. G., \& Fidell, L. S. (2014). Using Multivariate Statistics (6th Ed.). Harlow, UK: Pearson.

Tompkins, C. A., Bloise, C. G. R., Timko, M. L., \& Baumgaertner, A. (1994). Working memory and inference revision in brain damaged and normally aging adults. Journal of Speech Language and Hearing Research, 37, 896-896.

Tulsky, D. S., Carlozzi, N., Chiaravalloti, N. D., Beaumont, J. L., Kisala, P. A., Mungas, D., Conway, K., \& Gershon, R. (2014). NIH toolbox cognition battery (NIHTB-CB): List sorting test to measure working memory. Journal of the International Neuropsychological Society, 20, 599-610.

Vallat-Azouvi, C., Pradat-Diehl, P., \& Azouvi, P. (2012). The Working Memory Questionnaire: A scale to assess everyday life problems related to deficits of working memory in brain injured patients. Neuropsychological Rehabilitation, 22, 634-649.

Visser-Keizer, A. C., Meyboom-De Jong, B., Deelman, B. G., Berg, I. J., \& Gerritsen, M. J. J. (2002). Subjective changes in emotion, cognition, and behavior after stroke: Factors affecting the perception of patients and partners. Journal of Clinical and Experimental Neuropsychology, 24, 1032-1045.

Vogel, A. P., Maruff, P., \& Morgan, A. T. (2010). Evaluation of communication assessment practices during the acute stages post stroke. Journal of Evaluation in Clinical Practice, 16, 1183-1188.

Warrington, E. K., \& Shallice, T. (1969). The selective impairment of auditory-verbal short-term memory. Brain, 92, 885-896. 
Wechsler, D. (1981). Wechsler Adult Intelligence Scale - Revised. San Antonio, TX:

Psychological Corporation.

Wechsler, D. (1997). Wechsler Memory Scale - III. San Antonio, TX: Psychological Corporation.

Wechsler, D. (2000). WAIS-III: Manuel de l'Echelle d' Intelligence de Wechsler pour Adultes. Paris: Centre de Psychologie Appliquée.

World Health Organisation (2001). International Classification of Functioning, Disability and Health (ICF). Geneva: Word Health Organisation.

Zakariás, L., Keresztes, A., Marton, K., \& Wartenburger, I. (2016). Positive effects of a computerised working memory and executive function training on sentence comprehension in aphasia. Neuropsychological Rehabilitation, 21, 1-18. 
Table 1. Responses to survey questions regarding views about STM/WM assessment

\begin{tabular}{|c|c|c|c|c|c|}
\hline \multirow[t]{2}{*}{ Abbreviated Question } & \multirow{2}{*}{$\begin{array}{c}\text { Strongly } \\
\text { Agree }\end{array}$} & \multicolumn{3}{|c|}{ Neither Agree } & \multirow{2}{*}{$\begin{array}{l}\text { Strongly } \\
\text { Disagree }\end{array}$} \\
\hline & & Agree & nor Disagree & Disagree & \\
\hline 12. Effective for documenting treatment progress & $28 \%$ & $36 \%$ & $28 \%$ & $7 \%$ & $2 \%$ \\
\hline 13. Valuable when selecting treatment procedures & $43 \%$ & $41 \%$ & $12 \%$ & $3 \%$ & $0 \%$ \\
\hline 14. Valuable when making prognostic decisions & $31 \%$ & $43 \%$ & $19 \%$ & $6 \%$ & $0 \%$ \\
\hline 15. Would use STM/WM tests if they were more accessible & $26 \%$ & $36 \%$ & $24 \%$ & $13 \%$ & $1 \%$ \\
\hline 16. Would adopt STM/WM tests if their role in aphasia & $32 \%$ & $46 \%$ & $18 \%$ & $3 \%$ & $0 \%$ \\
\hline was well documented & & & & & \\
\hline
\end{tabular}

Note: The percentages in this table are based on 145 respondents. 
Table 2. Rankings of tests by popularity in terms of frequency of use and in terms of unavailability

\begin{tabular}{llll}
\hline & Most popular & Least popular & Unavailable \\
\hline $\mathbf{1}$ & CLQT (48\%) & Corsi Block (44\%) & WRAML (55\%) \\
$\mathbf{2}$ & CAT (39\%) & Corsi Tapping (43\%) & SCCAN (54\%) / TOMAL (54\%) \\
$\mathbf{3}$ & Token Test (36\%) & WMS (42\%) & \\
\hline
\end{tabular}

Notes: CLQT: Cognitive-Linguistic Quick Test; CAT: Comprehensive Aphasia Test; WMS:

Wechsler Memory Scale; WRAML: Wide Range Assessment of Memory \& Learning; SCCAN:

Scales of Cognitive \& Communicative Ability for Neurorehabilitation; TOMAL: Test of

Memory \& Language.

Table 3. Summary of regression analysis: clinicians vs. researchers/clinicians

\begin{tabular}{lccccc}
\hline & B & Std. Err. & Wald $\chi^{2}$ & p & Exp. (B) \\
\cline { 2 - 6 } Variables & & & & & \\
\hline Frequency of STM/WM & .024 & .233 & .011 & .918 & 1.024 \\
assessment (question 8) & & & & & \\
Factor 1 (questions 12, 13, 14) & .537 & .255 & 4.439 & $.035^{*}$ & 1.712 \\
Factor 2 (questions 15, 16) & .439 & .245 & 3.214 & .073 & 1.552 \\
Educational qualification & -3.169 & .531 & 35.645 & $.000^{*}$ & .042 \\
Experience in aphasia & .264 & .460 & .330 & .566 & 1.302 \\
\hline
\end{tabular}


Table 4. Summary of regression analysis: USA vs. UK

\section{B $\quad$ Std. Err. $\quad$ Wald $\chi^{2} \quad$ p $\quad$ Exp. (B)}

Variables

\begin{tabular}{lccccc}
\hline Frequency of STM/WM & .682 & .257 & 7.052 & $.008 *$ & 1.977 \\
assessment (question 8) & & & & & \\
Factor 1 (questions 12, 13, 14) & -.284 & .258 & 1.215 & .270 & .753 \\
Factor 2 (question 15, 16) & -.022 & .260 & .007 & .934 & .979 \\
Educational qualification & .571 & .754 & .573 & .449 & 1.769 \\
Experience in aphasia & -.131 & .506 & .067 & .796 & .878 \\
Clinician - researcher/clinician & -.163 & .762 & .046 & .830 & .849 \\
\hline
\end{tabular}




\section{Appendix A}

Survey Content

\section{Section 1: Demographic Information Characteristics}

(1) Would you describe yourself as: (Clinician; Clinician and Researcher; Researcher; Other, [please describe]); Please describe the clinical setting you work in: (Acute inpatient; Inpatient rehabilitation; Outpatient/community rehabilitation; Long-term care facility; Mixed role; Private practice)

(2) Are you based in? (US; Other country)

(3) What state are you based in? (List of the US states)

(4) In which country do you live? (List of countries)

(5) How many different people with aphasia do you assess a year, on average (estimated)? $(1-10 ; 11-20 ; 21-30 ; 31-40 ; 40+)$

(6) How many years of experience do you have working with people with aphasia?

(7) What is your highest earned degree? (Bachelors; Masters; Ph.D.; Clinical Doctorate; Other (please specify)

\section{Section 2: Mechanics of STM/WM Testing}

(8) I assess short term/working memory as part of providing services to people with aphasia. (Every time; Often; Sometimes; Seldom; Never)

(9) If you don't assess short term/working memory, is someone else in your clinical setting typically responsible for this task? (Yes; No) 
Psychologist (Neuropsychologist or Clinical Psychologist); Nurse; Occupational Therapist; Medical Doctor; Other health care professional) If you don't assess short term/working memory (and it is not assessed by other team members), please state the reasons you are not able to assess this cognitive ability. (Select all that apply) (Lack of time; Lack of access to tests; Available tests are not suitable for those with aphasia; Available tests outdated; Lack of training or experience with cognitive tests; Other, please specify)

\section{Section 3: Views towards STM/WM Testing}

I believe that assessing short term/working memory is effective for documenting progress in the treatment of people with aphasia. (Strongly Agree; Agree; Neither Agree or Disagree; Disagree; Strongly Disagree)

I believe that assessing short term/working memory is valuable when selecting treatment procedures for people with aphasia. (Strongly Agree; Agree; Neither Agree or Disagree; Disagree; Strongly Disagree)

I believe that assessing short term/working memory is valuable when making prognostic decisions about people with aphasia. (Strongly Agree; Agree; Neither Agree or Disagree; Disagree; Strongly Disagree) I would use short term/working memory tests more in aphasia management services if they were more accessible. (Strongly Agree; Agree; Neither Agree or Disagree; Disagree; Strongly Disagree) 
I would be willing to adopt short term/working memory if its role in managing aphasia was well documented. (Strongly Agree; Agree; Neither Agree or Disagree; Disagree; Strongly Disagree)

\section{Section 4: Frequency of Use of Specific STM/WM Tests}

How frequently do you use self-rating questionnaires about a person's subjective memory abilities when testing short term/working memory? (Every aphasia assessment; Only those with adequate reading/listening comprehension; Only those with a caregiver who can complete as a proxy; Never; Other (please specify))

If you assess short term/working memory as part of providing services to people with aphasia, please indicate how frequently you use the following measures (see Appendix 3 for list of tests): (Always; Sometimes; Never; Not Available to me, but I would use if I had access; Not available to me, but I wouldn't use even if I had access)

\section{Section 5: Preference of Assessment Tools}

Please rank our top three preferred STM/WM tests from the drop down lists provided with your most preferred first and your third most preferred last. (Drop down menus give the tests from \#19 as options)

Please rank the type of subtest for testing STM/WM. To do this, use your mouse to drag and drop the test types so that your most preferred is on the top $\left(1^{\text {st }}\right)$ position and your lease preferred as the last $\left(6^{\text {th }}\right)$ position. (Semantic Memory, Other (Please Specify), Digit Span, Tapping Span, Recall, List Learning)

\section{Final question of the survey:}

Please list any additional comments 


\section{Appendix B}

Information about the PCA analysis

\begin{tabular}{lllll}
\hline & Question 13 & Question 14 & Question 15 & Question 16 \\
\hline Question 12 & .560 & .575 & .371 & .243 \\
Question 13 & & .603 & .277 & .294 \\
Question 14 & & .343 & .224 \\
Question 15 & & & .514 \\
\hline
\end{tabular}




\section{Appendix C}

Rotated factor pattern and final communality estimates from PCA

\begin{tabular}{|c|c|c|c|}
\hline \multicolumn{2}{|c|}{ Factors } & \multirow[b]{2}{*}{$\mathrm{h}^{2}$} & \multirow[b]{2}{*}{ Abbreviated questions } \\
\hline 1 & 2 & & \\
\hline .849 & .146 & .96 & 12. Effective for documenting treatment progress \\
\hline .831 & .161 & .71 & 13. Valuable when selecting treatment procedures \\
\hline .811 & .201 & .74 & 14. Valuable when making prognostic decisions \\
\hline .109 & .880 & .73 & 15. Would use STM/WM tests if more accessible \\
\hline .249 & .822 & .78 & 16. Would adopt STM/WM tests if role was well documented \\
\hline
\end{tabular}




\section{Appendix D}

\section{STM/WM tests featured in the survey}

Corsi Blocks

Corsi Tapping Span

Token Test (De Renzi \& Vignolo, 1962) or Revised Token Test (McNeil \& Prescott, 1978)

Semantic Memory, Recognition Memory, Repetition of Digits Strings, Repetition of Sentences subtests of the Comprehensive Aphasia Test (Swinburn et al., 2005)

Story Retelling and Design Memory subtests of the Cognitive Linguistic Quick Test (HelmEstabrooks, 2001)

Auditory Digit Repetition, Auditory Digit Matching Span, Pointing Span for Noun-Verb, and Sentence Repetition subtests of the Psycholinguistic Assessment of Language Processing in Aphasia (Kay et al., 1992)

List Learning, Story Memory, Digit Span, and Coding subtests of the Repeatable Battery for the Assessment of Neuropsychological Status (Randolph, 2012)

Immediate Memory subtest of the Ross Information Processing Assessment (Ross-Swain, 1986)

Immediate Figure Recall of the Rey-Osterrieth Complex Figure Test (Rey, 1964)

Immediate Recall and Oral Expression I: Repetition \& Immediate Recall subtests of the Scales of Cognitive and Communicative Ability for Neurorehabilitation (Milman \& Holland, 2012)

Facial Memory, Memory for Stories, Object Recall, Visual Sequential Learning, Word List Learning, and Memory for Location subtests of the Test of Memory and Learning (Reynolds \& Bigler, 1994)

Digit Span and Letter-Number subtests of the Wechsler Adult Intelligence Scale (Wechsler, 2010)

Letter-Number Sequencing, Spatial/Tapping Span, Spatial Attention, Verbal Paired Associates, Logical Memory, Symbol Span, and Digit Span subtests of the Wechsler Memory Scale

(Wechsler, 2009)

Verbal Working Memory, Symbolic Working Memory, Story Memory, Picture Memory, Verbal Learning and Design Memory subtests of the Wide Range Assessment of Memory and Learning (Sheslow \& Adams, 2003) 


\section{Appendix E}

Written-in responses regarding "Other" STM/WM tests used

*Aachener Aphasie Test (Huber et al., 1983); Sentence Repetition subtest

*Arizona Battery for Communication of Dementia (Bayles \& Tomoeda, 1993)

Aphasie-Check Liste (Kalbe et al., 2002)

Airplane Test (Crovitz, 1979)

Aphasie-Schnell-Test (Kroker, 2006)

Barcelona Test (Pena, 1990)

Brief Cognitive Assessment Tool (Mansbach, 2012)

Bielefelder Aphasie Screening (Wittler \& Hielscher-Fastabend, 2006)

Bilingual Aphasia Test (Paradis, 1987)

Brief Visuospatial Memory Test - Revised (Benedict, 1997)

Burns Brief Inventory of Communication and Cognition (Burns, 1997)

California Verbal Learning Test (Delis et al., 2000)

Camden Memory Tests (Warrington, 1996)

Corsi Block Tapping (De Renzi \& Nichelli, 1975)

Hopkins Verbal Learning Test (Brandt \& Benedict, 2001)

Kaplan Baycrest Neurocognitive Assessment (Leach et al., 2000)

*Leiter International Performance Scale (Roid \& Miller, 2011); Visuo-Spatial Direct Memory or

Visuo-Spatial Reverse Memory subtests

*Montreal Cognitive Assessment (Nasreddine et. al., 2005)

Modified Mini Mental State Exam (Teng \& Chui, 1987)

**N-KAI (Letter Naming, Number Repetition, Letter Repetition)

A Developmental NEuroPSYchological Assessment (Korkman, Kirk, \& Kemp, 1998)

Norwegian Basic Assessment for Aphasia (Reinvang \& Engvik, 1980); Sentence Repetition subtest

Raven's Progressive Matrices (Raven, Raven, \& Court, 2003)

Rey Auditory Verbal Learning Test (Schmidt, 1996)

*Rivermead Behavioral Memory Test (Cockburn \& Smith, 1989); RBMT-III Line Drawing subtest 
Mottier Test (Risse \& Kiese-Himmel, 2009); Syllable Repetition subtest

Short Orientation Memory Concentration Test (Katzman et al., 1983)

Thematic Apperception Test (Murray, 1943)

Wechsler Memory Scale IV (Wechsler, 2009); Visual Working Memory Index

Informal/generic tests/tasks:

Face Recognition

Picture pointing span of spoken words

Informal word span, forward recall

Month and Digit Ordering

*Questions during interview with patient and family/informal

Self Created Number Lists

Self Created Words Lists

Semantic Memory

Story Memory

Verbal Learning

*Indicates test or task was written-in by 2 or 3 respondents. All others were written-in by only one participant.

** The participant did not provide sufficient information/description to find the citation for this test. 


\section{Appendix F}

Written-in responses regarding self-rating questionnaires

Bilingual Aphasia Test (Paradis, 1987)

Burden of Stroke Scale (Doyle et al., 2004)

Brief Cognitive Rating Scale (Reisberg \& Ferris, 1988)

Comprehensive Aphasia Test (Swinburn et al., 2005)

*Cognitive Failures Questionnaire (Broadbent et al., 1982)

*Dysexecutive Questionnaire (Wilson et al., 1996)

*Memory Self Efficacy Questionnaire (Berry \& West, 1989)

Memory Failures of Everyday Questionnaire (Carrasco, Pena, \& Sueiro, 2012)

Patient Competency Rating Scale (Prigatano et al., 1986)

Questionnaire of Auto-Evaluation of Memory (Van der Linden et al., 1989)

Rating Scale of Attentional Behavior (Pondsford \& Kinsella, 1991

**RICA

Stroke and Aphasia Quality of Life Scale-39 (Hilari et al., 2003)

Self Concept Scale (Robson, 1989)

Skills Assessment Inventory (Focus on Function Workbook; Klein \& Hahn, 2007)

**Sohlberg Metamemory Questionnaire

*The Brain Injury Workbook Questionnaire (Powell, 2013)

Working Memory Questionnaire (Vallat-Azouvi, Pradat-Diehl, \& Azouvi, 2012)

Token Test (De Renzi \& Vignolo, 1962) or Revised Token Test (McNeil \& Prescott, 1978)

*Proprietary/Own/Informal Self-Rating Questionnaire

Repetition of Sentences

*Indicates test or task was written-in by 2 or 3 respondents, except for the

Proprietary/Own/Informal response which was provided by 8 respondents. All others were written-in by only one participant.

** The participant did not provide sufficient information/description to find the citation for this measure. 